\title{
Science Based Stockpile Stewardship and RIA
}

\author{
L., Ahle, L. A. Bernstein, M. Hausmann, D. J. Vieira
}

\section{June 9, 2003}

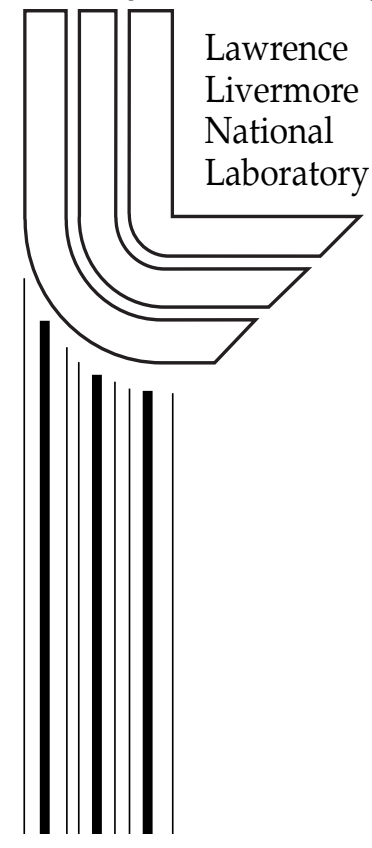


This document was prepared as an account of work sponsored by an agency of the United States Government. Neither the United States Government nor the University of California nor any of their employees, makes any warranty, express or implied, or assumes any legal liability or responsibility for the accuracy, completeness, or usefulness of any information, apparatus, product, or process disclosed, or represents that its use would not infringe privately owned rights. Reference herein to any specific commercial product, process, or service by trade name, trademark, manufacturer, or otherwise, does not necessarily constitute or imply its endorsement, recommendation, or favoring by the United States Government or the University of California. The views and opinions of authors expressed herein do not necessarily state or reflect those of the United States Government or the University of California, and shall not be used for advertising or product endorsement purposes. 


\title{
Science Based Stockpile Stewardship and RIA
}

\author{
Larry Ahle $^{1}$, Lee A. Bernstein ${ }^{1}$, Marc Hausmann ${ }^{2}$, and David J. Vieira ${ }^{2}$ \\ ${ }^{1}$ Lawrence Livermore National Laboratory, Livermore, California, 94538 \\ ${ }^{2}$ Los Alamos National Laboratory, Los Alamos, New Mexico, 87545
}

\begin{abstract}
One aspect of Science Based Stockpile Stewardship (SBSS) is to improve the quality of neutron cross section data for certain isotopes. The isotopes of interest are used to monitor neutron and charged particle fluxes in environments of brief, intense neutron fluxes. The accuracy of flux determination is dependent on the accuracy of cross section data for the stable isotopes loaded into the system and the unstable isotopes produced when the neutrons are incident on the monitor. For isotopes with a half-life greater than one day it is possible, given the production rates of RIA, to make radioactive targets for neutron irradiation. This would require the ability to harvest isotopes at RIA, an onsite radiochemistry facility for processing the harvested material into a target, and an onsite neutron source facility. The radiochemistry facility will need to handle activity levels on the order of 100's of Curie's while the neutron source facility will need to provide high intensity "monoenergetic" neutrons from 10's keV to $20 \mathrm{MeV}$. For isotopes with a half-life much less than one day, only indirect methods can be used to get information on the neutron cross sections because of the lack of a target. Both experimental techniques will be discussed with their impact on the infrastructure at RIA, as well as the general case for the interest of SBSS in RIA.
\end{abstract}

\section{INTRODUCTION}

The Science Based Stockpile Stewardship program (SBSS) insures the reliability and safety of the US nuclear weapon stockpile without testing. It relies on state of the art simulation capabilities whose inputs include nuclear cross sections with an emphasis on neutron reaction cross sections. Nuclear cross sections play their most important role when interpreting measurements of isotope production to determine neutron and charged particle fluxes in brief intense neutron flux environments. While many of the cross sections of interest for stable nuclei and a few unstable nuclei have been measured, most of the cross sections involving unstable nuclei are determined from theory alone. In addition to the measurements of nuclei used as flux monitors, neutron cross section measurements on prompt fission fragments near mass number 95 and 147 and the actinides uranium, neptunium, and plutonium are some of the other isotopes that play an important role in SBSS.

The Rare Isotope Accelerator (RIA) promises the ability to make a wide variety of isotopes throughout the chart of the nuclides ${ }^{1}$. Of most interest to the nuclear physics community are those nuclei far from stability that cannot be produced by any other facility. But RIA also promises never before achieved production rates of near stability nuclei. Both of these capabilities offer opportunities to make cross section measurements that were not possible before. For nuclei with a half-life of one day or more, targets can be made and irradiated with neutrons, while for isotopes with a half-life much less than a day indirect methods must be used. SBSS is therefore interested in RIA and developing the necessary infrastructure to allow the desired nuclear cross section measurements to occur.

\section{NEUTRON MONITORS}

As an example of how elements can be used as neutron flux monitors, assume that ${ }^{90} \mathrm{Zr}$ is added to the experimental environment. As neutrons react with the ${ }^{90} \mathrm{Zr}$ atoms, (n,2n) reactions will make ${ }^{89} \mathrm{Zr}$ and ${ }^{88} \mathrm{Zr}$ while competing $(\mathrm{n}, \gamma)$ reactions will reduce the amount of ${ }^{89} \mathrm{Zr}$ and ${ }^{88} \mathrm{Zr}$. ${ }^{89} \mathrm{Zr}$ has a 3.27 day half-life while ${ }^{88} \mathrm{Zr}$ has an 83.4 day half-life, implying both isotopes will exist for some time after the neutron flux event. By collecting zirconium afterward and using gamma ray spectroscopy, the ratio of ${ }^{88} \mathrm{Zr}$ to ${ }^{89} \mathrm{Zr}$ can be determined. This ratio can then be used to infer information about the neutron flux, provided the appropriate cross sections are known accurately.

In reality, the reaction networks involved are much more complicated than the simple example above. Figure 1 shows in more detail a reaction network starting from ${ }^{90} \mathrm{Zr}$. Notice that almost all of the reactions involve cross section measurements on unstable nuclei. Also, while there is good data for cross sections involving the ${ }^{90} \mathrm{Zr}$ ground state, there is very little data for the other cross sections. Note metastable stable states also play a role. For many of the neutron flux monitors, the cross sections on stable nuclei have been measured to 5\% and having this level of accuracy for some of the more important unstable nuclei is desired. Table 1 lists some of the elements used as particle flux 
monitors. Isotopes and isomers with the given mass range and with a half-life longer than a day are of particular interest. Note that all of the nuclei are near stability and most are proton rich.

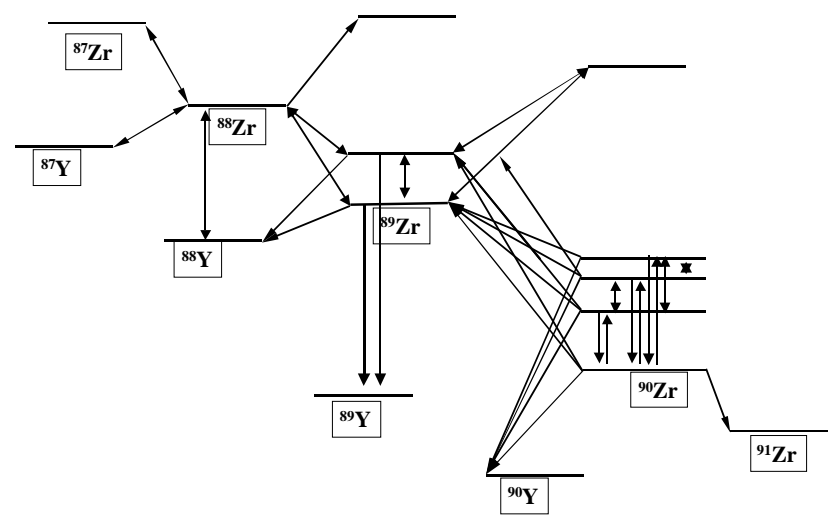

Figure 1. Reaction network for zirconium. Only ${ }^{89} \mathrm{Y},{ }^{90} \mathrm{Zr}$, ${ }^{91} \mathrm{Zr}$ have a stable ground state.

Table I

A list of some of the elements used as particle flux monitors and the mass ranges for which cross section measurements are important for SBSS.

\begin{tabular}{|c|c|c|c|}
\hline $\mathrm{Z}$ & $\mathrm{A}$ & $\mathrm{Z}$ & $\mathrm{A}$ \\
\hline $\mathrm{Kr}$ & $78-82$ & $\mathrm{Lu}$ & $170-178$ \\
\hline $\mathrm{Y}$ & $86-92$ & $\mathrm{Ta}$ & $179-184$ \\
\hline $\mathrm{Zr}$ & $87-90$ & $\mathrm{Ir}$ & $188-195$ \\
\hline $\mathrm{Nb}$ & $91-94$ & $\mathrm{Au}$ & $193-199$ \\
\hline $\mathrm{Eu}$ & $146-155$ & $\mathrm{Bi}$ & $204-210$ \\
\hline $\mathrm{Tm}$ & $166-171$ & $\mathrm{Th}$ & $230-234$ \\
\hline
\end{tabular}

\section{MEASUREMENT TECHNIQUES}

Figure 2 is a guide on how to do neutron cross-section measurements. The guide divides the parameter space of half-life versus atomic number into different measurement techniques and assumes a production rate of $10^{11-12} \mathrm{pps}$ and a neutron source capable of delivering $10^{10-11}$ neutrons/s on target. RIA can achieve such production rates for near stability nuclei. This figure is only to serve as a general guide. The best method for any given nucleus will depend on the details of that specific nucleus and the desired reaction. Also, the dashed lines indicate the boundaries are only approximate. Note from the chart that $\mathrm{Z}=92$ is a hard cut off and isotopes with a half-life less than one millisecond will be hard even for RIA to provide.

For a nuclei with a half-life of about one day and longer, it is possible to consider making sufficient material to form a target and perform direct neutron cross sections measurements. It is possible for low mass nuclei to be made in sufficient quantities before RIA using light ion direct reactions, such as $(\mathrm{p}, \mathrm{n})$. This is especially true on the proton rich side. For high mass nuclei, the number of stable and near stable isotopes complicates the direct reaction method of target production. For a half-life shorter than one day, it becomes increasingly more difficult to produce enough material, such that indirect methods need to be invoked in order to gain insight into the neutron cross sections.

Figure 2. General guide to neutron cross section measurement methods. Dashed lines indicate that boundaries are approximate. See text for details about direct and indirect measurements.

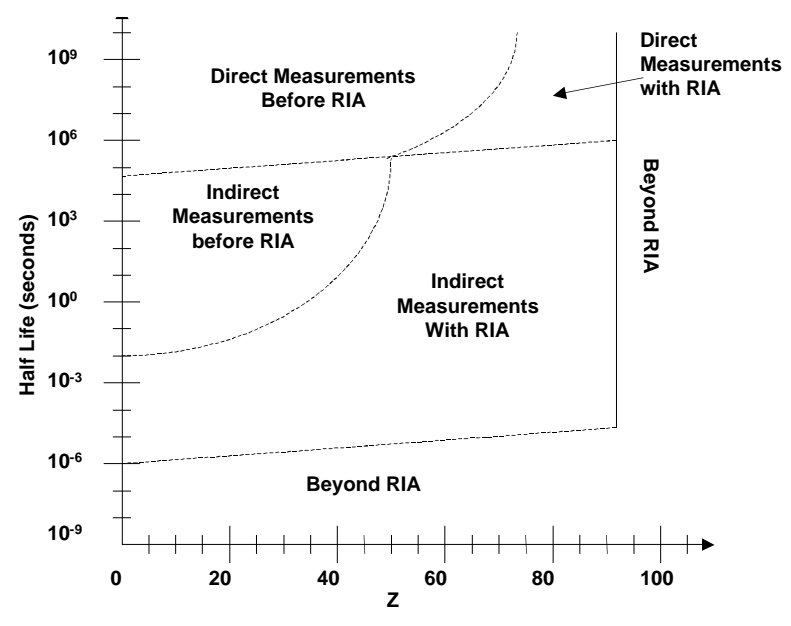

III.A. Direct Methods

There are two basic approaches to measuring neutron cross sections via neutron irradiation on targets. In the delayed method the target is irradiated with neutrons and afterwards radiation from the decaying reaction products are detected. This only works if the reaction product, or one of its daughter's lives long enough to enable counting. If a decay daughter is used, it is possible to do chemistry after the irradiation. This would be especially desirable if the target is radioactive, since this would separate the background radiation of the target from the reaction products. One of the most difficult issues to overcome for the direct method is target purity. Given a neutron flux of $10^{11}$ neutrons/second on target and an irradiation time of 1-2 days, then only 1 in $10^{9}$ target atoms will undergo the desired nuclear reaction of interest. Thus, the target must be free of the reaction product at that level, otherwise the experimental signal will be swamped by background. And if the interest is in $(n, 2 n)$ and $(n, \gamma)$ reactions as is the case for SBSS, then chemistry can not help purify the target, since the atomic number does not change. Therefore, either the production method must not produce this background or mass separation is required to obtain the appropriate purity. Reference 2 is an example of such a delayed measurement. 
The other approach to directly measure neutron cross sections is a prompt experiment that measures each nuclear reaction as it occurs. The choice of technique depends on the exact nature of the reaction of interest. In all cases, the prompt approach greatly reduces the requirement on target purity to the level of $10^{-2}$ or better, but now the detector must be able to handle the radiation coming from the target. Also, the detector must be shielded from the neutron source, usually requiring the radioactive target to be some distance away from the neutron source. This reduces the intensity of neutrons on target. It is important to maximize neutrons on target since this will reduce the amount of target material needed and thus reduce the background seen by the detector.

For $(n, 2 n)$ reactions, measuring the emitted neutrons is one way to avoid the gamma background. This approach was pioneered $\mathrm{J}$. Frehaut ${ }^{3}$ in measuring many stable and actinide (n,2n) cross sections. This technique surrounds the target with a liquid scintillator loaded with gadolinium. Neutrons scatter in the liquid scintillator until they are captured by the gadolinium, releasing gamma rays in the process. These gammas are then detected by one of several phototubues. The overall efficiency for neutrons can be made quite high, near $80 \%$ for the scintillator ball used in reference 3. Shielding and enhanced segmentation will be needed to handle the high gamma background from the target.

For $(n, \gamma)$ reactions gamma rays must be detected which makes dealing with the gamma ray background from the target challenging. One way to approach this problem is to do total gamma ray calorimetry instead of looking at individual gamma rays. $\mathrm{DANCE}^{4}$ is a detector built to do just that. It is $\mathrm{a} \mathrm{BaF}_{2}$ array that deals with target background through segmentation and analysis tools to distinguish between multiple gamma rays emitted following neutron capture and low multiplicity gamma rays from radioactive target. In some cases shielding can be used to reduce low energy $(<200 \mathrm{keV})$ gamma rays from the decaying target.. Presently, a one-milligram target, which corresponds to a hundred day half-life $(\sim 1 \mathrm{Ci})$, is required because of the neutron source intensity at the Lujan Center/LANSCE.

For reactions that have a charged particle in the output, such as (n,p), (n, $\alpha),(n, n p)$, and (n,fission), detecting the charged particle offers an option to avoid the high gamma ray background. In this case, some sort of particle spectrometer would be needed and the charge particle detector would need to be shielded from the target. The target would also sit inside a magnetic field, which would bend the escaping charge particle around the shielding to the charge particle detector. The overall efficiency of this system will be low because of the geometrical acceptance to the detector, but it should still be high enough to allow a measurement. Much more development work is needed on this technique.

\section{III.B. Indirect Methods}

For nuclei with a short half-life, indirect methods become the more attractive experimental method. At the very least this will involve making mass, life-time, and structure measurements to give theory the best input possible in making accurate calculations of the appropriate reaction cross section. Level schemes will play a particularly important role in this avenue of approach. The RIA community is already planning these types of measurements.

The surrogate reaction technique, however, is one experimental method that offers the possibility of obtaining at least some cross section information for short-lived nuclei. The idea behind the surrogate approach is to look at another reaction that generates the same compound system as would be produced by the neutron reaction. One then measures the probability for the compound system to decay into the desired output channel. This probability when multiplied by the total neutron absorption cross section allows one to obtain the neutron cross section. This idea was first explored by Cramer and Britt ${ }^{5}$ and Britt and Wilhemy ${ }^{6}$ to infer $(n, f)$ cross sections from $(t, p f)$ and $\left({ }^{3} \mathrm{He}, \mathrm{xf}\right)$ reactions, respectively. The results from these initial attempts were good but there were still some significant disagreement between these reactions and direct $(\mathrm{n}, \mathrm{f})$ data when it was available. A recent reanalysis by Younes and $\mathrm{Britt}^{7}$ of the (t,pf) reactions incorporating spin and parity dependences into the data analysis achieved significantly better agreement. Currently, LLNL is trying to extend this approach to $(n, 2 n)$ and $(n, \gamma)$ reactions. While initial results are encouraging more analysis, experiments, and theory development are needed to understand the full utility of the surrogate approach.

\section{PERFORMING MEASUREMENTS AT RIA}

For indirect measurements, most if not the entire required infrastructure is already being developed by the RIA community. This is also true for surrogate reaction technique. Surrogate reaction experiments at RIA will utilize radioactive beams in inverse kinematics reactions. A gamma array will be important and charged particle detectors will also be needed. In some cases a recoil separator would also prove valuable. All of these pieces are already being planned by the RIA community, especially by the astrophysics community who are interested in $(n, \gamma)$ reactions for $\mathrm{s}, \mathrm{r}$, and rp process investigations.

Direct cross section measurements on the other hand, have facility requirements that are not necessarily being pursued by the RIA community. First, a method for collecting isotopes must be incorporated into the design of RIA. Once collected, the material must be processed into a target at a radiochemistry facility. Afterward, the target must be transported to a neutron source facility for irradiation. And if the delayed measurement method is used, it may be necessary to reprocess the target chemically to separate out the reaction products of interest. A low 
background counting facility would also be needed, regardless of the measurement method.

\section{IV.A. Harvesting Isotopes}

The Isotope Separation On-Line ${ }^{1}$ (ISOL) beam line offers two advantages for isotope harvesting, large production rates, up to $10^{12} \mathrm{pps}$, and low energy beams, typically $60 \mathrm{keV}$. The high production rates are important to minimize the half-life limit for making a target. The low energy allows easy collection by implantation in a foil since the nuclei will stop quickly without inducing unwanted nuclear reactions in the foil that would increase the impurity level. The disadvantage of the ISOL method is the chemistry dependence of the production rate. The ISOL method relies on release of the desired product from an amorphous material at high temperatures and then efficient ionization. Elements like noble gases release well, but elements with a high melting temperature like zirconium, do not.

Assuming a good release and ionization of the desired product, there are two locations where it might be possible to collect separated isotopes. Depending on the ion source used in the ISOL target, a large number of different isotopes will be extracted from the ISOL system. Mass separation is then applied in two stages to purify the ion beam. The preseparator, which does a rough separation, is one location where collection can occur. It is unclear whether the purity that can be obtained at this stage would be sufficient. For prompt measurements, it probably would be sufficient, but for delayed measurements, it probably would not be. After this low-resolution mass separator, a high-resolution mass separator is then used. This would give much better target purities. Both locations offer the possibility of collecting isotopes parasitically to other experiments, but to achieve the highest data purity samples, the collection would have to be done as a primary user of one of the ISOL production lines.

The fragmentation line overcomes the chemical limitation of the ISOL line, but at the cost of lower production rates and higher beam energy. The production in the fragmentation target can still be quite high, around $10^{11}$ pps, for lighter mass beams where the driver linac can deliver on the order of 100's of particle-microamps. But for heavy mass beams $(A>100)$, the beam current is down to 10 's of particle-microamps and as little as 1 particlemicroamp for a uranium beam. Some of this is due to the limitations of the ion source for the driver linac but beam heating in the production target is also limiting.

The nuclei of interest are produced as beam fragments and thus have a similar energy as the production beam, up to $350 \mathrm{MeV} /$ nucleon. This creates a challenge in stopping the ions for collection. The gas-stopping cell currently being developed uses electric fields to quickly guide ions with a half-life under one second to another ion source for reacceleration ${ }^{1}$. Thus, the beam current in the cell must be kept low so that plasma formation and space charge does not alter the electric fields. This is presently believed to keep the output of the gas cell down to $10^{9} \mathrm{pps}$. This limitation however may be overcome for target production since one is interested in much longer-lived isotopes. Complicating this issue is the nuclear reactions the ions will undergo as they are stopped. This will increase the impurities in the collected sample. Exactly how this might be accomplished will require further development.

Another issue that may prevent collection of isotopes all together from the fragmentation line is the stopping of the primary beam. The primary beam after it leaves the target will still be at least $30 \mathrm{~kW}$ of power and must be stopped in a controlled manner. Presently it is planned to stop the beam before the first quadrupole and not allow the primary beam to be transported through the mass separator. This will require the primary beam to be significantly deflected away from the desired isotopes very earlier in the separator. Thus, near stability isotopes may not make it all the way through the separator and may not be separated enough from the primary beam to allow collection near the beginning of the separator. Further study of these issues needs to be done.

\section{IV.B. Radiochemistry Facility}

At the heart of creating a target for neutron bombardment is radiochemistry. A $10 \mu \mathrm{g}$ sample of a 1 day half-life isotope implies about $10 \mathrm{Ci}$ of activity. The minimum amount of material needed will depend on the reaction rate, the measurement method, and the desired measurement accuracy. In most cases $10 \mu \mathrm{g}$ should be enough to perform measurements, though sometimes less material maybe required. But there is also the possibility that other radioactive species will be collected in the sample. Thus, a radiochemistry lab will need to be designed to handle a hundred Curie sample of gamma radiation. Hot cells capable of handling $1 \mathrm{kCi}$ of gamma radiation are not uncommon. These hot cells would allow chemistry to be performed to purify the collected sample and form a target suitable for neutron irradiation.

Transportation to and from the radiochemistry lab is another important issue. Presently, transportation from the collection area to the radiochemistry laboratory will be done above ground via some lead container. Having some sort of underground transport system for this part has been considered, but given the different possible locations of isotope collection some means of above ground transport will be needed. It is planned to have an underground transportation system from the radiochemistry laboratory to the neutron irradiation areas. This system is currently envisioned to be simply a cart on rails attached on both sides by a cable. The cable is then mechanically pulled in either direction to move the cart back and forth. There must also be a low background counting room near this facility to assay the radiochemical prepared targets. 


\section{IV.C. Neutron Source}

A separate but collocated mono-energetic neutron source is the final piece of infrastructure required for direct measurements. The range of neutron energies of interest starts from 10's keV and extends up to $20 \mathrm{MeV}$. In order to achieve this energy range, a mono-energetic but tunable neutron source maybe used. Table 2 lists various important neutron production reactions over the energy range of interest. All of these reactions involve the acceleration of protons, which could be accelerated as molecular hydrogen, and deuterons. It is also important to maximize the neutron flux on target because of the short half-life of the target. Thus a low energy intense light ion accelerator is required.

Figure 3 is a preliminary design of what a neutron source facility might look like. It has two accelerators, a 3 MeV Dynamitron ${ }^{8}$ and a $40 \mathrm{MeV}$ linac. The Dynamitron can accelerate high currents, up to 10's of milliamps and at an energy down to $50 \mathrm{keV}$. As designed, the Dynamitron delivers a DC current, but beam choppers have been with a Dynamitron to deliver a pulsed beam. The linac would start with a $2 \mathrm{MeV}$ RFQ and then continues to accelerate with DTL's up to the desired energy. The DTL's allow complete energy flexibility between 2 and $40 \mathrm{MeV}$. The expected beam current would be only several hundred microamps, which together with the lower energy range, are the reasons for the Dynamitron. The figure also shows three experimental areas. One of the areas is for low energy neutrons and the other two are for high-energy neutrons with beam stops for the deuteron beam. Two areas for radiochemistry are also shown in the figure. The underground transport system is not shown.

Table II

A list of various neutron production reactions.

\begin{tabular}{|c|c|c|}
\hline Reaction & Q value & Best Neutron Energy \\
\hline${ }^{7} \mathrm{Li}(\mathrm{p}, \mathrm{n}){ }^{7} \mathrm{Be}$ & $-1.64 \mathrm{MeV}$ & $0.1-0.4 \mathrm{MeV}$ \\
${ }^{3} \mathrm{H}(\mathrm{p}, \mathrm{n}){ }^{3} \mathrm{He}$ & $-0.76 \mathrm{MeV}$ & $0.1-3 \mathrm{MeV}$ \\
${ }^{2} \mathrm{H}\left({ }^{2} \mathrm{H}, \mathrm{n}\right){ }^{3} \mathrm{He}$ & $3.27 \mathrm{MeV}$ & $3-10 \mathrm{MeV}$ \\
$\mathrm{X}\left({ }^{2} \mathrm{H}, \mathrm{pn}\right) \mathrm{X}$ & $-2.25 \mathrm{MeV}$ & $>6 \mathrm{MeV}$ \\
${ }^{3} \mathrm{H}\left({ }^{2} \mathrm{H}, \mathrm{n}\right){ }^{4} \mathrm{He}$ & $17.59 \mathrm{MeV}$ & $13-16 \mathrm{MeV}$ \\
\hline
\end{tabular}

As mentioned above, maximizing neutron flux is an important goal of the facility. A neutron flux as high as $10^{11}$ neutrons per second on target has been assumed in this paper. The final neutron flux achieved will be a trade off involving target issues and neutron energy spread. This is especially true at the lower energies, in which the proton or deuteron beam stops quickly thus broadening the energy distribution of the neutrons. For neutron energies below $300 \mathrm{keV}$, it is quite practical to use time of flight techniques to determine neutron energy, thus eliminating the need for a monoenergetic neutron source in this energy range. This would simply require a pulsed neutron source and flight paths on the order of one meter ${ }^{9}$. The final neutron flux will also depend on the production method as different methods have different reaction cross-sections and different degrees of kinematic focusing. More work is needed on the neutron source design and a second iteration of the design is planned over the next six months.

\section{SUMMARY}

The Science Based Stockpile Stewardship Program is interested in neutron cross sections on many unstable nuclei. The Rare Isotope Accelerator promises the capability of producing unstable isotopes in sufficient quantities allowing neutron cross section measurements. Thus, the stockpile stewardship community is very interested in RIA and insuring the correct infrastructure is present to allow direct neutron measurements. These include the ability to collect the appropriate isotope, process the material into to a target at a radiochemistry facility, and irradiate the target with neutrons at a separate but collocated neutron source. The final specification of these pieces are still being developed and we are working with the rest of the RIA community to develop these ideas.

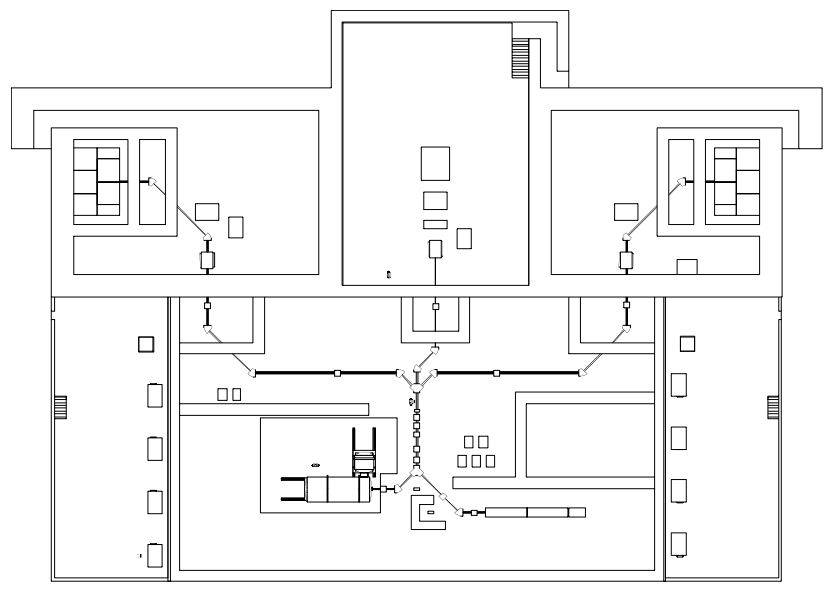

Figure 3. A drawing of a possible design for a neutron production facility at RIA. The experimental areas are up top, with a low energy neutron area in the middle. The two room on either side at the bottom are areas for radiochemistry. The dimensions of the entire facility are approximately $80 \times 60 \mathrm{~m}$. See text for other details.

\section{ACKNOWLEDGEMENTS}

This work was performed under the auspices of the U.S. Department of Energy by the University of California, Lawrence Livermore National Laboratory under contract No. W-7405-Eng-48 and Los Alamos National Laboratory under contract W-7405-ENG-36. We would also like to thank B. Rusnak and R. Berio for their contribution in the neutron source facility design and to Mark Stoyer and Ken Moody for their information on the radiochemistry. 


\section{REFERENCES}

1. "ISOL Task Force Report to NSAC", H. Grunder et al. Eds., November 1999, available on internet at $\langle$ http://srfsrv.jlab.org/isol/ISOLTaskForceReport.pdf $\rangle$.

2. R. J. PRESTWOOD, K. W. THOMAS, D. R. NETHAWAY, and N. L. SMITH, "Measurement of 14$\mathrm{MeV}$ Neutron Cross Sections for ${ }^{88} \mathrm{Zr}$ and ${ }^{88} \mathrm{Y}$ ", Phys. Rev. C, 29, 3, 805 (1984).

3. J. FREHAUT, "Use of the Large Gadolinium-Loaded Liquid Scintiallator Technique for $(n, 2 n)$ and $(n, 3 n)$ Cross Section Measurements", Nuc. Instr. And Meth., 135, 511 (1976).

4. M. HEIL et al., "A $4 \pi \mathrm{BaF}_{2}$ Detector for (n, $\gamma$ ) CrossSection Measurements at a Spallation Neutron Source", Nuc. Inst. And Meth. A, 459, 229 (2001).

5. J. D. CRAMER and H. C. BRITT, "Neutron Fission Cross Sections for ${ }^{231} \mathrm{Th},{ }^{233} \mathrm{Th},{ }^{235} \mathrm{U},{ }^{237} \mathrm{U},{ }^{239} \mathrm{U},{ }^{241} \mathrm{Pu}$, and

${ }^{243} \mathrm{Pu}$ from 0.5 to $2.25 \mathrm{MeV}$ Using (t,pf) Reactions", Nuc. Sci and Eng., 41, 177 (1970).

6. H. C. BRITT and J. B. WILHELMY, "Simulated Cross Sections for Exotic Actinide Nuclei”, Nuc. Sci. and Eng., 72, 222 (1979).

7. W. YOUNES and H. C. BRITT, "Neutron-induced Fission Cross Sections Simulated from (t,pf) Results", Phys. Rev. C, 67, 024610 (2003).

8. S. MATSUYAMA et al., "A Compact PostAcceleration Beam Chopper for a 4.5 MeV Dynamitron Pulsed-Neutron Generator", Nuc. Inst. And Meth. A, 348, 34 (1994).

9. R. Reifarth, R. C. Haight, F. Kappeler, D. J. Vieira, "Neutron Studies Below $1 \mathrm{MeV}$ at RIA", These Proceedings. 